\title{
New Systematic Energy Generation Having Superconductivity Properties without Refrigeration
}

\author{
Author Details \\ Name: Shinichi Ishiguri \\ Affiliation: Nihon University \\ 1-2-1, Izumi-Cho, Narashino City, Chiba, 275-8575 JAPAN \\ TEL: +81-47-474-9143 \\ Email: ishiguri.shinichi@nihon-u.ac.jp / superconductivity01@docomo.ne.jp
}

\begin{abstract}
This paper proposes a method of extracting energy from zero-point energy and evaluates the amount of energy gained. In addition, this electric circuit-based approach exhibits the Meissner effect, suggesting a new type of superconductivity that does not require refrigeration. The proposed method can provide extremely large amounts of energy, which is more than a conventional power station, without consuming fossil fuels or emitting radiation. Thus, it has the potential to solve the global energy problem. It involves preparing two electric loops containing diodes and connecting the loops together with current sources. The diodes are oriented in the same direction within each loop but in opposite directions in different loops. With this setup, the currents from the current sources build iteratively within the loops, resulting in large output currents. Our numerical analysis indicates that extremely large electric potentials are produced, which in turn yield large output currents. In addition, we confirm numerically that the voltage is zero around a loop and show analytically that the Meissner effect is present, proving the existence of a new type of superconductivity. Furthermore, when we introduce induction coils to not break the loop's symmetry, they store extremely large amounts of energy and we can thus obtain energy from them via discharge currents.
\end{abstract}

Keywords: current source, diode, divergent current density, new superconductivity type, zero voltage, symmetric electric circuit, Meissner effect, electric potential, vector potential, Lorentz conservation law

\section{Introduction}

Herein, we describe a new source of large amounts of energy and investigate it via numerical analysis. The proposed method involves two electric circuit loops with diodes linked by current sources. With this setup, we obtain an apparently divergent current density, meaning that the currents iteratively build within the loops, yielding extremely large DC currents. In addition, if we introduce induction coils not to break the circuit's symmetry, they can store large amounts of energy and discharge it in the form of 
large DC currents.

The design of this system was motivated by the current global energy problems. There are several issues with combustion-based power stations. First, the supply of fossil fuels is finite and, due to the IT revolution, the demand for electric power is increasing. The resulting fossil fuel consumption is raising global temperatures. In addition, the fuel for such power stations is mainly obtained from the Middle East, which is politically unstable.

To limit the use of combustion-based power generation, Japan is considering the increased use of atomic power. However, it has previously suffered from extremely serious earthquakes and tsunamis, which have destroyed an atomic power station and caused radiation leakage. In short, we should not become totally dependent on unstable atomic power stations.

Turning to existing natural energy sources such as solar cells [1] generates relatively little power and depends on the weather, which means that the amount of power generated is variable. Therefore, in Japan, such natural energy sources can only augment the main power station network in a limited way, and cannot replace combustion-based or atomic power generation.

In addition, as long as we depend on combustion-based and atomic power generation, electric power generation must be concentrated in certain locations and thus we must employ a power transmission system, which inevitably results in significant energy losses. In Japan, this system is also rendered unstable due to frequent earthquakes. We therefore need to employ distributed energy generation in every home.

This paper proposes a novel method of generating energy in a laboratory setting. We estimate that this can produce more energy than combustion-based or atomic power generation. In addition, it does not require fossil fuel or generate radiation. It is also inexpensive to construct, with the only significant expense being the current sources (which would currently cost about 100,000 yen).

The principle behind our approach also enables us to predict, via numerical and analytical results, that it exhibits a new type of superconductivity which is not tied to specific substances or temperatures. Conventional superconductors have previously been investigated [2-6], and we have published several articles concerning a new type of superconductivity that does not require refrigeration [7-9]. The underlying principle is that, by employing doped semiconductors, the applied electrostatic fields cancel the ohmic fields (i.e., ohmic voltages) when we supply a current from a current source. With this setup, the total internal voltage is zero but the current is non-zero due to carrier diffusion, which is unrelated to any macroscopic electric field.

In our previous articles, we demonstrated that pairs of electrons can be combined by the spin magnetic field at extremely small relative distances $(1 \AA)$ and, by considering each pair's complete center-of-mass motion, we found that Bose-Einstein condensation occurs at critical currents. In addition, we demonstrated the theoretical predictions via experiments showing zero electric resistance and the Meissner effect. This type of superconductivity is not related to specific materials and does 
not require refrigeration, but the small critical currents involved prevent the use of large power supplies. However, the system discussed in the present paper differs from our previous approach in that it enables the generation of extremely large DC currents.

First, we introduce the main principle behind our work. Next, we analyze it numerically and discuss the results. Then, we discuss the source of the energy produced, as well as showing that the two electric loops in the proposed device enable it to exhibit superconductivity without refrigeration.

\section{Principle}

Fig. 1 illustrates the principle underlying this study. Here we see two electric loops, each involving four normal diodes oriented in the same direction. The electric loops in (a) and (b) each have four junctions, by which they are combined via four current sources, as shown in Fig. 2.

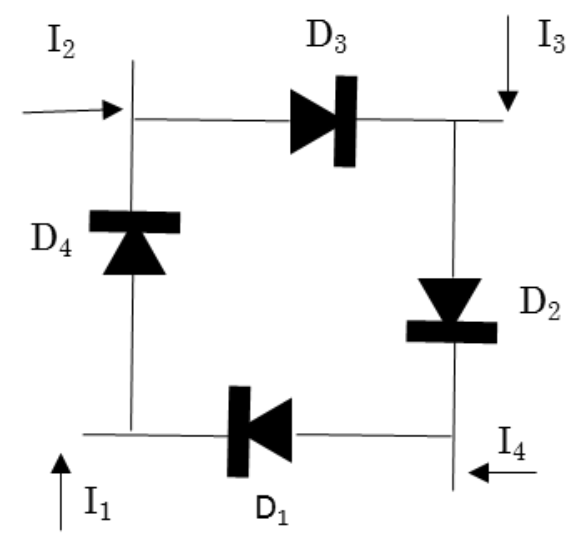

(a)

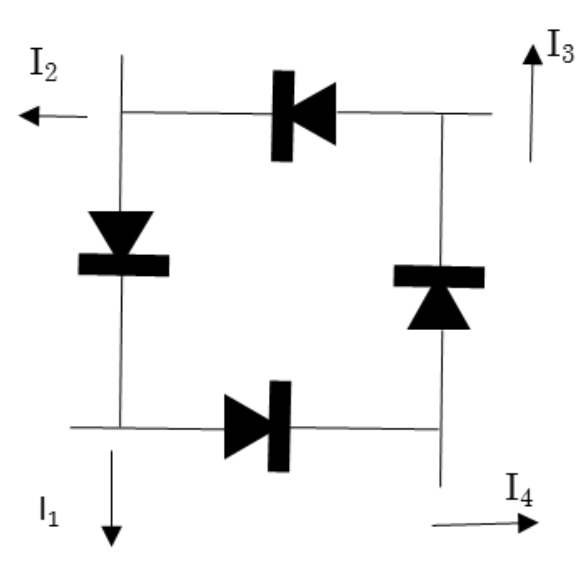

(b)

Fig.1 Overview of the proposed system, based on two electric loops. In each loop, the four diodes are oriented in the same direction, but the directions in (a) and (b) are opposite. The loops are connected via current sources. For example, the currents $I_{1}$ in (a) and (b) are connected via a current source. The current inputs along the loop in (a) build iteratively around the loop, resulting in a large output current. 


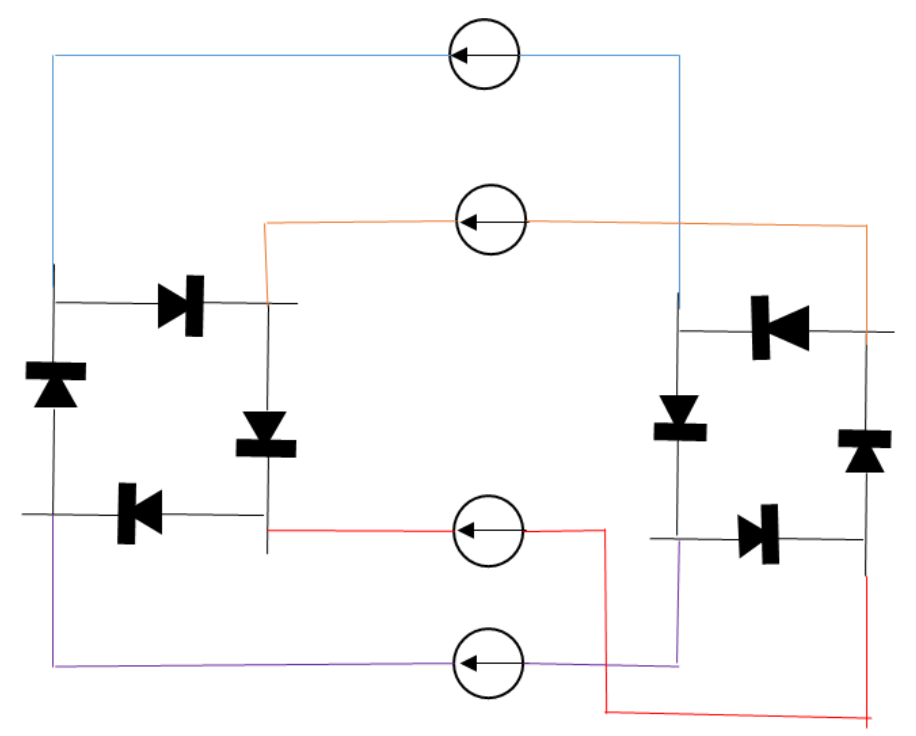

Fig.2 Illustration showing how the two electrical loops in Fig. 1 are connected by current sources. Taken together with Fig. 1, this demonstrates the principle behind the system.

To understand the principle behind our scheme, consider Fig. 1. In (a), current $I_{1}$ enters the circuit, passes through diode $\mathrm{D}_{4}$ and then combines with current $I_{2}$. Together, these currents pass through diode $\mathrm{D}_{3}$ and further combine with current $I_{3}$. Then, this current goes on to combine with current $I_{4}$ before completing the loop and combining again with currents $I_{1}, I_{2}$, and so on. Continuing this process results in the generation of an extremely large current.

If we apply Kirchhoff's voltage law to Fig. 1(a), we find that the total voltage over the diodes is zero, i.e.,

$4 V_{D}=0$, (1)

where $V_{\mathrm{D}}$ denotes the diode voltage. Given that the currents through the diodes are non-zero, this implies that each diode satisfies a superconductivity condition. Later, we use simulations to show that, indeed, the diode voltages are zero but the currents are non-zero.

Now, we need to describe the characteristics of current sources. Electric circuit sources can be divided into two main types, namely voltage and current sources. Voltage sources supply a constant voltage to their taps under a load, meaning that the current varies according to Ohm's law. On the other hand, current sources, as shown in Fig. 3, must supply a constant current to their taps under a load, meaning that the voltage varies according to Ohm's law. Current sources are commonly used in electrical and semiconductor technologies and are made up of transistors, whose collector currents represent internal current sources when we consider their equivalent circuits [10]. 


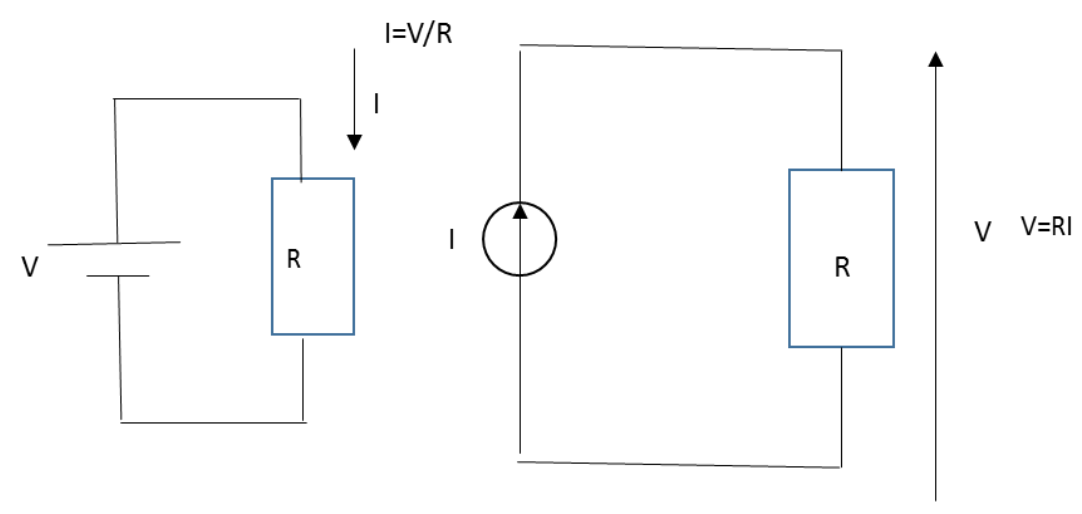

(a)

(b)

Fig. 3. Simple comparison between voltage and current sources. The voltage source in (a) provides a constant voltage, regardless of load, so the current varies depending on the load. Generally, voltage sources have almost zero internal resistance. In contrast, the current source in (b) provides a constant current, regardless of load, so the voltage varies depending on the load. The arrow indicates the direction of the current it provides, which can be produced by utilizing the collector currents of NPN transistors. Note that current sources have almost infinite internal resistance.

\section{Method}

To simulate the circuit shown in Figs. 1 and 2, we employed the PSIM software package (Powersim Inc, USA). Since the electrical circuit loops in Fig. 1 involve a superconductivity condition, we did not use current and voltage measurements because these would essentially be related to the devices' internal electric resistances. In addition, we assumed the diodes and current sources to be ideal, meaning that the current sources' internal impedances were infinite. For these reasons, we instead measured the electric potentials (i.e., electromagnetic scalar potentials) using PSIM's probes.

\section{Results}

Fig. 4 illustrates one of the diode loops, where we have introduced currents of $1 \mu \mathrm{A}$ and probes V11-V14 measure the electrostatic potentials. The results, shown in Fig. 5, highlight the following two important facts.

1) Extremely large electric potentials were produced. As discussed in Section 5.1, this implies the presence of extremely large currents. For comparison, Figs. 6(a) and (b) show an ordinary electrical circuit and the corresponding electric potential. Comparing these two results emphasizes 
just how large the electrical potentials seen in Fig. 5 are.

2) Probes V11-V14 all measured identical electric potentials. If we consider Kirchhoff's voltage law, this implies that the voltage around the loop is zero and the superconductivity condition is satisfied.

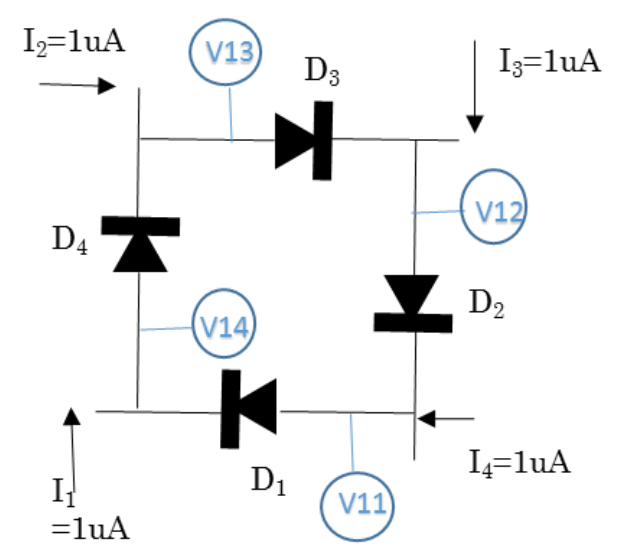

Fig. 4. Diode loop with a $1 \mu \mathrm{A}$ input. Here, four probes for measuring the electric potentials (V11V14) are located symmetrically around the loop.

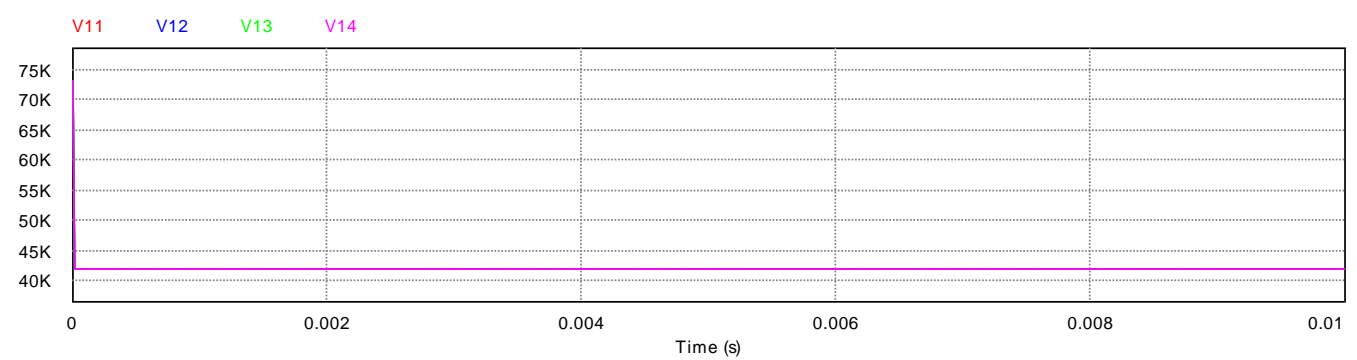

Fig. 5 Results for the setup shown in Fig. 4, with the probe voltage shown on the $y$-axis. Probes at four points around the loop were used to measure the electric potentials, and these results show that all probes measured identical potentials. This implies that, around the loop, the voltages summed to zero and hence the superconductivity condition was satisfied. The extremely large electric potentials imply the presence of a large DC current. 


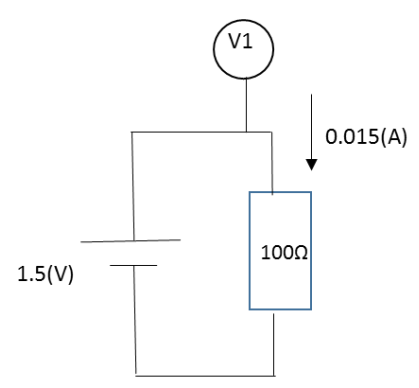

(a)

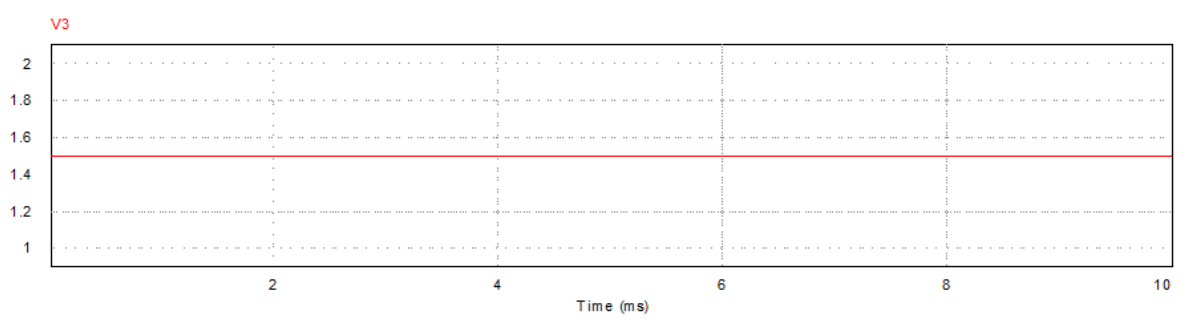

(b)

Fig. 6 (a) Conventional electric circuit, presented for comparison. In (b), the probe voltage is shown on the $y$-axis. Here, the electric potential is around $1 \mathrm{~V}$. Comparing this with the potentials seen in our system (Fig. 5) shows just how large those potentials are.

As shown in Fig. 7, we also considered the case of $1 \mathrm{~A}$ inputs. Here, we also introduced four $1 \mathrm{H}$ inductance coils in order to examine whether the coils were able to store energy. The results, shown in Fig. 8, imply that the superconductivity condition was still satisfied even when we introduced these four (superconducting) coils, and that the extremely large electric potentials were still present. As will be discussed in Section 5.1, these large potentials indicate extremely large DC currents. Thus, the coils must be storing extremely large amounts of energy. In Section 5.1, we estimate just how much energy they are storing. Note that it is important for all four superconducting coils to be discharged simultaneously when the energy is needed, in order to maintain their superconductivity. 


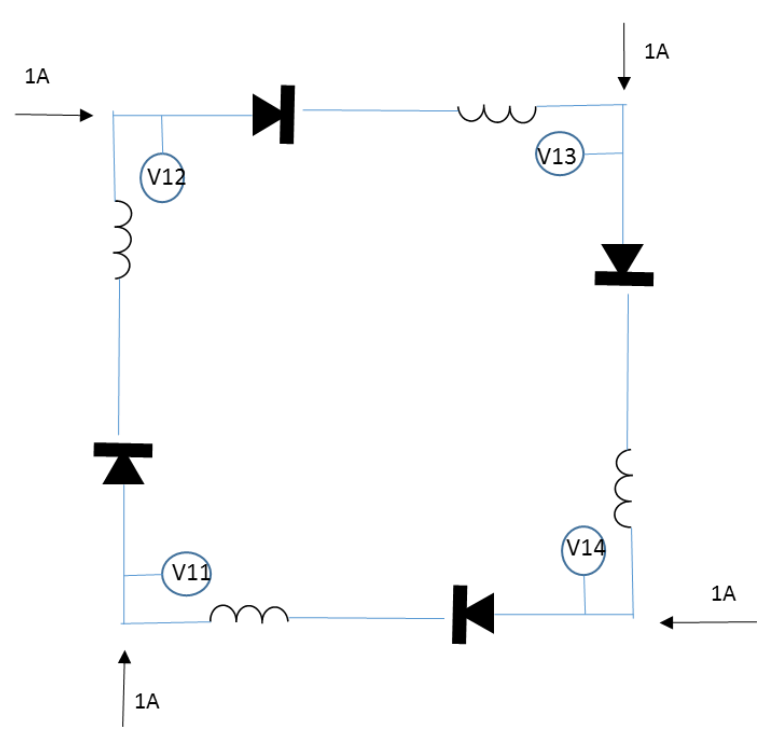

Fig. 7 Diode loop with $1 \mathrm{~A}$ inputs and four $1 \mathrm{H}$ induction coils. These coils must be placed symmetrically in order to maintain the superconductivity condition. The four electric potential probes are likewise connected symmetrically for the same reason.

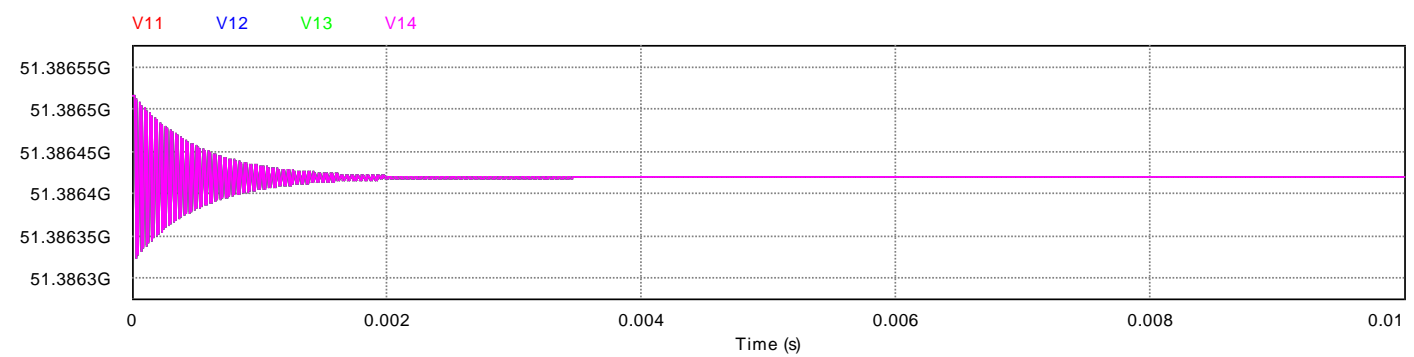

Fig. 8 Results for the setup shown in Fig. 7, with four $1 \mathrm{H}$ induction coils and $1 \mathrm{~A}$ inputs. The probe voltage is shown on the $y$-axis. After some initial fluctuations, the four electric potentials converge to $51.3 \mathrm{GV}$ after $0.002 \mathrm{~s}$. This implies that, even after introducing the coils, the electric potentials are still extremely high, implying that the superconductivity condition is still satisfied. Thus, we believe that these coils can store extremely large energies.

\section{Discussion}

\subsection{Output current and energy stored}


To transform an electric potential into a DC current, we can employ the following energy conservation rule:

$q \varphi=\frac{1}{2} m v^{2},(2)$

where $q, \varphi, m$, and $v$ denote the electric charge, electric potential, electron mass, and drift velocity, respectively. From this equation, we can derive the carriers' drift velocity as

$v=\sqrt{\frac{2 q \varphi}{m}} \cdot(3)$

Thus, we can express the current density as

$j=q n v=q n \sqrt{\frac{2 q \varphi}{m}}$,

where $n$ denotes the carrier concentration.

For a $1 \mu \mathrm{A}$ input, the resulting electric potential is approximately $\varphi \approx 45 \mathrm{kV}$. For comparison, the conventional electric circuit in Fig. 6 yields an electric potential of $\varphi_{0} \approx 1 \mathrm{~V}$. Since we used the same numerical software for both calculations, the current leads must have identical cross sections. Thus, we obtain following ratio for the current densities:

$\frac{j}{j_{0}}=\sqrt{\frac{\varphi}{\varphi_{0}}}$

where $j$ and $j_{0}$ denote the output current densities for our system and the conventional circuit, respectively. Since the current $I_{0}$ in the conventional circuit is simply $I_{0}=0.015 \mathrm{~A}$, we can calculate the current in our system as

$I=\sqrt{\frac{\varphi}{\varphi_{0}}} I_{0}=3.15$ A. (6)

This implies that the tiny $1 \mu \mathrm{A}$ input current produces a $3.15 \mathrm{~A}$ output current.

Based on Fig. 8, we can calculate the output current and the energy stored in the coils. The current calculation, following the method used above for the $1 \mu \mathrm{A}$ input case, yields an output current of $3 \mathrm{kA}$ and $18 \mathrm{MJ}$ of energy stored in the four coils. This is more energy than many conventional power stations can produce.

\subsection{Use of electric potential probes}

Next, we should explain why we employed electric potential probes rather than ammeters or voltmeters. As is well-known, such meters have two taps (compared with a single tap for an electric potential probe) and internal electrical resistances. If we had employed them in our system, their resistances would potentially have become zero, and ammeters and voltmeters can generally only function when their electrical resistances are non-zero. Thus, ammeters would not have worked in the PSIM simulation. In addition, if we had connected four voltmeters to the diodes in our system's electric 
circuit loop, not all of the voltmeters would have displayed zero voltages because in fact the system exhibits extremely large electric potentials, i.e., the current is non-zero. This implies that some of the voltmeters would have detected voltages due to the current passing through their internal resistances. Consequently, the voltmeters would have shown positive and negative voltages so as to satisfy Kirchhoff's voltage law, implying that current was flowing around the loop in different directions and violating the laws of electromagnetism. In Section 5.3, we will demonstrate the existence of a current around the system's loop. In summary, we had to employ probes to measure the electric potential in this study.

\subsection{Existence of a current around the diode loop}

According to the laws of electromagnetism, an electric potential is related to a static electric field, as follows:

$\varphi=-E_{\theta} \xi$, (7)

where $E_{\theta}$ and $\xi$ denote the electrostatic field along a loop and an arbitrary small positive constant. In our system, we have the following relation for each loop:

$\oint \varphi d s \neq 0$. (8)

Thus,

$\oint \varphi d s=-r \xi \int E_{\theta} d \theta \neq 0$, (9)

where $r$ denotes the average loop radius. Since the constant $\xi$ is non-zero, satisfying eq. (9) requires the following:

$\oint E_{\theta} d \theta \neq 0 .(10)$

Consequently, the average electric field is non-zero:

$<E_{\theta}>\neq 0$. (11)

This implies that we can consider an average drift velocity along the electric loop of

$<\mathrm{v}>=\mu\left\langle E_{\theta}>\right.$,

where $\mu$ denotes the electron mobility. Thus, the current density around the loop is

$\left.j=\sigma<E_{\theta}\right\rangle=q n<v>\neq 0$, (13)

where $\sigma$ denotes the conductivity. This demonstrates that there is a rotational current around the loop.

\subsection{Superconductive energy generation}

Now, let us consider why we are able to obtain energy, based on the conservation of energy. Lorentz's conservation law is initially as follows $[11,12]$ :

$\left|A_{0}\right|^{2}-\left(\frac{\varphi_{0}}{c}\right)^{2}=\left(\frac{1}{q c} \frac{1}{2} \hbar \omega\right)^{2},(14)$ 
where $A_{0}, \varphi_{0}$, and $c$ denote the initial vector and electric potentials and the speed of light, respectively. In the steady state, the zero-point energy $(1 / 2 \hbar \omega)$ of the vacuum on the right-hand side of eq. (14) is consumed, resulting in the terms on the left-hand side increasing. In other words, the vector and electric potentials increase due to energy conservation (strictly speaking, to maintain the Lorentz conservation law). Thus,

$|A|^{2}-\left(\frac{\varphi}{c}\right)^{2}=0,(15-1)$

where $A$ and $\varphi$ denote the final vector and electric potentials. In other words,

$A=\frac{\varphi}{c},(15-2)$

where

$\mathrm{A} \gg A_{0,} \varphi \gg \varphi_{0} .(16)$

In addition, we can use eq. (15-2) to demonstrate the Meissner effect, as follows. From eq. (7), we have

$j=\sigma E_{\theta}=-\frac{\varphi}{\xi} \sigma=-\frac{c \sigma}{\xi} A .(20)$

This implies that the London equation holds, which is important because our system exhibits superconductivity not only in terms of the transport properties but also the magnetic properties. In addition, gage symmetry is broken [13]. Thus, we can conclude the currents we see are essentially superconducting currents that do not require refrigeration.

Any researchers attempting to reproduce these experiments in practice will need to be very careful to observe suitable safety protocols, due to the extremely large electric potentials involved. In particular, the expert assistance of electrical system engineers would likely be helpful.

\section{Conclusion}

In this study, we aimed to obtain high stored energy densities in an ordinary laboratory setting. First, we created two electrical circuit loops containing diodes, with the diodes oriented in the same direction within each loop but in opposite directions in different loops. Then, we connected the two loops together with current sources. In this novel device, the currents from the current sources add together iteratively around each loop, and are never subtracted, resulting in extremely large output currents.

We then estimated the output current based on the electric potential. By applying Kirchhoff's voltage law to one loop, we found that it satisfied a superconductivity condition. When we then introduced four induction coils while maintaining symmetry, the coils also satisfied the same superconductivity condition. For a $1 \mathrm{~A}$ input, the four coils stored around $18 \mathrm{MJ}$ of energy due to the extremely large output current. Note that, when attempting to obtain the coils' stored energy, they must be discharged simultaneously so as to maintain the superconductivity condition. 
In addition, we have shown analytically that the Meissner effect occurs, by demonstrating that the London equation holds and gage symmetry is broken.

The results in this paper are significant because they potentially enable us to obtain more energy than many power stations can produce in an ordinary laboratory setting, without consuming fossil fuels or producing radiation. This could contribute to preventing global warming. In addition, demonstrating a new type of superconductivity is also important from a physics viewpoint.

\section{References}

[1] R. Willams, The Journal of Chemical Physics 32 (5), 1505-1514 (1960)

[2] S. Uchida, Japanese Applied Physics (Institute Journal), Vol. 80, No. 5, (2011) 383-386

[3] T. Fujita, J. Cryogenics and Superconducting Societies of Japan, Vol. 47, No. 2, (2012) 89-95

[4] P. A. Lee, et al, Rev. Modern Phys., Vol. 78, (2006) 17

[5] M. Ogata, H. Fukuyama, Rep. Prog. Phys., Vol. 71, (2008)

[6] M. Azuma, et al, Phys. Rev. Lett, 733463 (1994)

[7] S. Ishiguri, J. Supercond. Nov. Magn. 24, 455 (2011)

[8] S. Ishiguri, Int. J. of Mod. Phys. B, 27, 1350045 (2013)

[9]S. Ishiguri, Preprints 2018, 2018110636 (doi: 10.20944/preprints201811.0636.v1),(2018)

[10] N. Fuji, Analog electron circuit, p.57, p.119 Shokodo Tokyo, (1984)

[11] S.Ishiguri,, Preprints 2018, 2018120194 (doi: 10.20944/preprints201812.0194.v1),(2018)

[12] Y. hara, Elementary Particle Physics, p.54 Shokabo Tokyo (2003)

[13] H. Aoki, Introduction of Superconductor, p.72 Shokabo Tokyo, (2010)

\section{Acknowledge}

We thank Enago (www.enago.jp) for English language Review.

\section{Additional information}

This paper is not related to any competing interests such as funding, employment and personal financial interesting. Moreover, this paper is not related to non-financial competing interesting 